\title{
MicroRNAs from Diagnosis to Therapy: Future Perspective
}

\author{
Abdul Hafeez Kandhro
}

Faculty of Medical Technology, Center of Data Mining and Biomedical Informatics, Mahidol University, Thailand

${ }^{*}$ Corresponding author: Abdul Hafeez Kandhro, Faculty of Medical Technology, Center of Data Mining and Biomedical Informatics, Mahidol University, Salaya, Phutthamonthon, Nakhon Pathom, Thailand, Tel: +66850243948; E-mail: abdul.haf@student.mahidol.ac.th; hafeezjaan77@yahoo.com

Received date: October 03, 2016; Accepted date: October 12, 2016; Published date: October 26, 2016

Copyright: (c) 2016 Kandhro AH. This is an open-access article distributed under the terms of the Creative Commons Attribution License, which permits unrestricted use, distribution, and reproduction in any medium, provided the original author and source are credited.

Citation: Kandhro AH. MicroRNAs from diagnosis to therapy: Future perspective. Transl Biomed. 2016, 7:4.

\section{MicroRNA Therapeutic Challenges: Future Perspective}

MicroRNAs (miRNAs) are emerged as posttranscriptional regulators, involved in many biological processes including, cell cycle, differentiation, proliferation, migration, secretion, aging and apoptosis, essential for the development and physiology of various organs [1]. Dysregulation of miRNAs may alter gene networks in disease states such as, metabolic diseases, cancers, neurodegenerative diseases; thus, miRNA therapy could restore gene expression in the cells to reverse back in normal state. Their dysregulation may lead to the development of potential therapeutic targets for treating various diseases [2]. Furthermore, a single miRNA can target single or multiple mRNA gene targets [3]. Promising preclinical studies in the biomedical/clinical field demonstrated constructive therapeutic strategy for treating disorders ranging from metabolic diseases, cancers, neurodegenerative disorders to organ failure, although several challenges related to tissue specificity, targeted delivery remain to be overcome. Moreover, ratio of successful experimental studies is low, due to expensive technologies and approaches used in miRNA discovery, identification of biomarkers and therapeutic markers. Until now, miRNAs have been extensively studying, but their specific functions remain largely unknown [4].

The emergence of miRNAs has garnered in physiological processes, functions as a regulator and controlling metabolic homeostasis has recently raised in miRNAs research, biomarker discovery and designing therapeutic markers. The future perspective of miRNAs as important regulators of metabolism/pathways has greater concern not only from a scientific standpoint, but also from a clinical perspective. Although, complexity of miRNA-mRNA interactions, detection methodologies, and technical difficulties has been arises, especially in vivo [5]. Recent efficacy data from reported studies of animals and humans indicate potential, completely new class of drug such as, anti-miR therapy that inhibits specific miRNAs. Many advantages of miRNAs (as therapeutic point of view) such as, miRNAs are small size contains a known sequence which is often conserved completely among species, an attractive feature from a drug development perspective [6].
Currently, four types of RNA therapeutics are under clinical development and pre-clinical/clinical phase trials, including anti-sense oligonucleotides (ASO), small interfering RNA (siRNA) and microRNA mimetics (miRNA-mimic) and antimiRNA oligonucleotide inhibitors (AMO) [2]. Methods for selectively inhibiting miRNA function in vivo through the delivery of inhibitory oligonucleotides have raised hopes for new therapeutic modalities for treating disease. However, despite these advances, many questions remain regarding the mechanistic basis of miRNA actions, and numerous issues be addressed on the path toward development of miRNA-based therapeutics [6]. Several recent reviews have provided indepth discussion of the roles for individual miRNAs in the development of tissue/organ and disease [6]. As miRNAs may target multiple different mRNAs, thus they may regulate a same or similar pathway, which provides a unique ability for anti-miRNA therapeutics to regulate complex physiologic functions [7]. However, challenges raise concerns over miRNA potential off-target effects that might be reduce by designing in vivo model experimental work [8]. Anti-miRNA have delayed onset of actions, which may take several days in contrast to classical pharmacological agents. However, anti-miRNAs actions are long-lived, due to their higher stability and intracellular deposition from which they may slowly release [6]. Summarizing the facts that dysregulation of miRNAs often contributes to disease progression, therapeutic inhibition of these regulatory molecules has the potential to improve disease outcomes without disrupting normal physiologic functions.

\section{Future Perspective}

Nevertheless, still need to be more understanding about the precise mechanism of miRNA specific function and action within complex disease pathways and as a regulator could be, modulate in chronic disease with tissue-specific manner. We could learn deeply with systems biology approaches, to describe completely the role of miRNA and activity under conditions of homeostasis and disease. More understanding and elucidating potentiality of miRNAs needed from the recent available miRNA profiling data obtained from human disease, combined with sensitive, accurate, and flexible detection techniques such as, small RNA sequencing, quantitative PCR 
methods for circulating blood miRNAs and analysis of complex data sets by powerful bioinformatics/biostatistical analysis. These steps could bring potential result in further clarification of miRNA-mediated pathways in diseases. The use of miRNA as the future targeted therapy is too bright and impulsive at this moment; nevertheless, with rapidly increasing number of miRNA discoveries since more than 10 years, is certainly promising and hopeful.

\section{References}

Ha TY (2011) MicroRNAs in human diseases: from cancer to cardiovascular disease. Immune Netw 11: 135-154.

Hennessy EJ, KJ Moore (2013) Using microRNA as an alternative treatment for hyperlipidemia and cardiovascular disease: cardiomiRs in the pipeline. J Cardiovasc Pharmacol 62: 247.

3. Valinezhad Orang A, Safaralizadeh R, Kazemzadeh-Bavili $M$ (2014) Mechanisms of miRNA-mediated gene regulation from common downregulation to mRNA-specific upregulation. Int J Genomics 2014: 970607.

Samanta S, Balasubramanian S, Rajasingh S, Patel U, Dhanasekaran A, et al. (2016) MicroRNA: A new therapeutic strategy for cardiovascular diseases. Trends Cardiovas Med.

5. Iorio MV, Croce CM (2012) MicroRNA dysregulation in cancer: diagnostics, monitoring and therapeutics. A comprehensive review. EMBO Mol Med 4: 143-159.

Olson EN (2014) MicroRNAs as therapeutic targets and biomarkers of cardiovascular disease. Sci Transl Med 6: 3.

Xu J, Zhao J, Evan G, Xiao C, Cheng Y, et al. (2012) Circulating microRNAs: novel biomarkers for cardiovascular diseases. J Mol Med 90: 865-875.

Matsui M, Corey DR (2016) Non-coding RNAs as drug targets. Nat Rev Drug Discov. 